\title{
PS-CDMA: Spreding Signals for CDMA Designed in the Frequency Domain using Perfect Sequences
}

\author{
E. Del Re, R. Fantacci and L. S. Ronga. \\ LENST Laboratory - E.E.Dept - University of Florence \\ Via di Santa Marta 3, 50139 FLORENCE, Italy
}

\begin{abstract}
In this paper are described some new approaches to the definition of CDMA spreading functions. The simplest method increases the code space up to $50 \%$. The most sophisticated one performs a very strong rejection to the delayed replicas of the interfering signals. Several advantages have also been found in the FFT implementation of PS-CDMA transmitters and receivers, the elimination of the analog matched filter being the most attractive one. Theoretical results have been supported by computer simulations, and comparisons with the traditional DS-CDMA design of spreading functions are also included.
\end{abstract}

\section{Introduction}

The DS-CDMA technique relies on an efficient design of the spreading codes, with a small cross-correlation index to permit the retrieval of the desired user's information, and an autocorrelation shape as closer as possible to the Dirac's delta; this to avoid unexpected auto-interference effects due to the reception of delayed version of the transmitted signal.

In some CDMA applications, however, the standard design in the time domain of the spreading codes may result inappropriate, especially in presence of multi-path where time-shifted replicas of the same signal interfere with the desired one.

The basic theory of the Digital Signal Processing suggests an alternative method to design spreading functions and the resulting technique has some interesting properties which may result useful in the presence of multi-path environments.

In this work we show how the spreading sequences can be alternatively designed in the frequency domain. The flat spectral occupancy, the auto-correlation and other interesting properties are highlighted, and some examples of applications are given in order to compare this design technique with the standard one.

\section{The Phases Sequence}

In band limited channels the spectral design is the preferred technique for defining signals tp be used in digital communications. The spectral design however has some attractive properties that may result useful even in the design of wide spectrum channels. Consider a class of real baseband signals defined as

$$
\begin{aligned}
s_{i}(t) & =\sqrt{\frac{2}{T_{b}}} \sum_{k=1}^{K}\left[a_{i}(k) \cos \left(\frac{2 \pi k t}{T_{b}}\right)\right. \\
& \left.+b_{i}(k) \sin \left(\frac{2 \pi k t}{T_{b}}\right)\right]
\end{aligned}
$$

where

$s_{i}(t)$ is the signal assigned to user $i$ in a communication system where the channel is shared among $U$ users; $1 \leq i \leq U$.

$T_{b}$ is the time length of the bit.

$a_{i}(k)$ and $b_{i}(k)$ are the real taps of the $k$ - $t h$ spectral component of the signal $i$.

Note that the parameter $K$ determines the amount of spectral components of the signal so it influences directly the spectral occupancy of the signal itself. Since the bit rate is related only to the parameter $T_{b}$ and the spectral lines of the continuous extension of the signal are integer multiples of $\frac{1}{T_{b}}$, we can refer to $K$ as the spreading factor of the resulting CDMA system.

Note also that if the system appears in a time window of length $T_{b}$, his spectrum has exactly $2 K+1$ degrees of freedom. We consider signals whose mean value over the bit interval is zero, thus they can be described by the $2 K$ real taps as defined before.

Directly from the definition in (1) we can derive the energy value of the signal in a symbol interval

$$
\mathcal{E}_{i}=\int_{-T_{b} / 2}^{T_{b} / 2} s_{i}^{2}(t) d t=\sum_{k=1}^{K}\left[a_{i}^{2}(k)+b_{i}^{2}(k)\right]
$$

The cross-correlation for two signals is defined as

$$
\begin{aligned}
\left\langle s_{i}(t), s_{j}(t)\right\rangle & =\int_{-T_{b} / 2}^{T_{b} / 2} s_{i}(t) s_{j}(t) d t \\
& =\sum_{k=1}^{K}\left(a_{i}(k) a_{j}(k)+b_{i}(k) b_{j}(k)\right)
\end{aligned}
$$



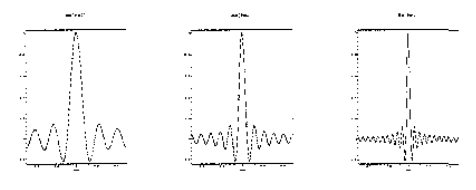
(a) $K=$
(b) $K=$
10
(c) $K=$
20

Figure 1: Auto-correlation index $T_{b}=1$

The autocorrelation measure is related to the ability of a matched filter in a receiver to reject delayed versions of the incoming desired signal. Let us consider the autocorrelation function for the class of signal defined by (1). A relevant measure for this is the following

$$
\begin{aligned}
\psi_{i}\left(\tau, K, T_{b}\right) & =\frac{1}{K} \int_{-T_{b} / 2}^{T_{b} / 2} s_{i}(t) s_{i}(t-\tau) d t \\
& =\frac{1}{K} \sum_{k=1}^{K}\left(a_{i}^{2}(k)+b_{i}^{2}(k)\right) \cos \left(2 \pi k \tau / T_{b}\right)
\end{aligned}
$$

If a flat spectral occupancy is desired, the following rela tion is imposed to the spectral coefficients:

$$
a_{i}^{2}(k)+b_{i}^{2}(k)=1 \quad \forall k
$$

With the previous relation auto-correlation measure results:

$$
\psi_{i}\left(\tau, K, T_{b}\right)=\sum_{k=1}^{K} \cos \left(2 \pi k \tau / T_{b}\right)
$$

In figure 1 are plotted the values of $\psi$ for some values of the spreading factor $K$.

As can be seen by the graphs, the larger is $\mathrm{K}$, the narrower is the auto-correlation peak. The height of the first non-center peak is constant relatively to the the height of the center peak as $\mathrm{K}$ increases. This may result useful for the development of an algorithm for the phase recovery based on training sequences. The value of the quantity $e(\tau)=1-\psi(\tau)$ may be used as an error function in a interval centered in 0 , whose local minimization leads to the exact bit synchronization.

It should also be noted that the:

$$
\lim _{K \rightarrow \infty} \frac{\psi_{i}\left(\tau, K, T_{b}\right)}{K}= \begin{cases}1 & \text { for } \tau=0 \\ 0 & \text { for } \tau \neq 0\end{cases}
$$

which is the ideal behavior of the auto-correlation function.

\section{The sequences factory}

In order to allow several users to transmit over the same portion of the spectrum we must provide a unique code for each of them. In this section is shown a method to build the user codes.

If the desired value of $K$ is even, the vector $\mathbf{c}$, defined as

$$
\mathbf{c}_{i} \equiv\left[a_{i}(1), b_{i}(1), a_{i}(2), b_{i}(2), \ldots a_{i}(K), b_{i}(K)\right]
$$

is built from the following binary set of values:

$$
\{1 / \sqrt{2 K},-1 / \sqrt{2 \widetilde{K}}\}
$$

Any traditional technique for orthogonal codes can be applied without modification (Walsh, etc.).

If an odd value for $K$ is requested, the phase sequences signals may be modified as follows:

$$
s_{i}^{*}(t)=s_{i}(t)+\sqrt{\frac{2}{T_{b}}} a_{i}(0)
$$

where $a_{i}(0)$ is chosen among the values in the set:

$$
\{1 / \sqrt{K+1},-1 / \sqrt{K+1}\}
$$

while the coefficients $a_{i}(k)$ and $b_{i}(k)$, with $k=1, K$, are chosen from the set

$$
\{1 / \sqrt{2(K+1)},-1 / \sqrt{2(K+1)}\}
$$

It is straightforward to prove that the cross correlation property holds. The code vector defined in (7) has now an additional element: $a_{i}(0)$. This permits the use of evenlength codes, such as Gold codes, cyclic, $\mathrm{BCH}$, etc.

All the above signals are designed with unitary energy.

\section{Polyphase Sequences}

In order to fully take advantage of the unique structure of the proposed class of signals, in this section the polyphase sequences are introduced.

We first introduce an alternate notation for the signals defined by (1). A complex representation of the spreading signals is considered.

We can rewrite the definition of the signals as follows:

$$
s_{i}(t)=\sqrt{\frac{2}{T_{b}}} \sum_{k=1}^{K} S_{i}(k) e^{j 2 \pi k t / T_{b}}
$$

Where $S_{i}(k)$ is a complex-valued spectral coefficient.

In order to generate signals with flat amplitude spectrum and unitary energy, the values of $S_{i}(k)$ are chosen with constant modulus and variable phase, as follows:

$$
S_{i}(k)=\frac{1}{\sqrt{K}} e^{j \theta_{i}(k)} \quad \forall k, \forall i
$$

We now introduce the concept of polyphase sequences as a generalization of the design method described in section 3 . 


\section{Perfect N-Phase Sequences}

A polyphase or N-Phase sequence is a sequence whose elements are of the form $\exp (j 2 \pi n / N)$, with $0 \leq n<N$.

If $r(x)$ and $q(x)$ are two $\mathrm{N}$-Phase sequences of length L, the Periodic Cross-correlation Function (PCCF) is defined as:

$$
\varphi_{r q}(l)=\sum_{x=0}^{L-1} r^{*}(x) q((x+l) \quad \bmod L) \quad 0 \leq l<L
$$

The respective autocorrelation function is obtained if the sequence $r(x)$ is replaced by $q(x)$.

The sequence $r(x)$ is called perfect if the side-lobes of the corresponding autocorrelation function are 0 .

In the past a large number of methods have been developed for searching or generating perfect N-Phase sequences. The basic theory of perfect sequences generation is due to Frank, Chu and Milewski $[3,2,4]$. Synthesis methods able to generate new sequences of various length from the basic ones,are also known [1].

The interest in this class of multi-phase sequences derives from the expression of the cross-correlation factor between two PS-CDMA signals defined by (9). In facts we have:

$$
\begin{aligned}
\left\langle s_{i}(t), s_{j}(t)\right\rangle & =\int_{-T_{b} / 2}^{T_{b} / 2} s_{i}(t) s_{j}(t) d t \\
& =\sum_{k=1}^{K}\left(a_{i}(k) a_{j}(k)+b_{i}(k) b_{j}(k)\right) \\
& =\operatorname{Re}\left\{\sum_{k=1}^{K} S_{i}^{*}(k) S_{j}(k)\right\}
\end{aligned}
$$

Equation (12) shows that the cross-correlation between two different signals is the real part of the complex crosscorrelation between the two sequences of the respective spectral coefficients. This suggests that the perfect sequences can be used as the spectral coefficients in PSCDMA signal definition.

\section{Perfect Arrays}

We have focused our attention on the so called perfect arrays, whose definition follows:

$$
\begin{aligned}
& s(x, y)=\exp \left\{j \frac{2 \pi}{N}(x \cdot y)\right\}, \\
& 0 \leq x<N, \quad 0 \leq y<N
\end{aligned}
$$

In this case we are able to generate $\mathrm{N}$ different sequences of length $\mathrm{N}$. It can be easily shown that each sequence is perfectly orthogonal to any other. The number of codes generated equals the spreading factor, as in the case of direct sequences derived from the Walsh's matrix.
By increasing $\mathrm{N}$ in one dimension only we progressively increase the number of codes with the same bandwidth occupancy. Of course the perfect cross-correlation property is lost but the cross-correlation values are still acceptable and are localized in the diagonal of the crosscorrelation matrix.

In the picture 2, are shown the cross-correlation indexes for each couple of generated codes. The coordinates of each pixel $(i, j)$ indicate the indexes of the considered codes, while the color of the pixel $c(i, j)$ is mapped to the following quantity:

$$
c(i, j)=\left|\operatorname{Re}\left\{\mathbf{S}_{\mathbf{i}}^{\mathbf{H}} \cdot \mathbf{S}_{\mathbf{j}}\right\}\right|
$$

where the superscript $H$ indicates the Hermitian operator.

In fig 2(I) is shown the case of 32 different codes of length (spreading factor) 32 . The orthogonality of the resulting signature waveforms is perfect and the cross correlation matrix is the identity matrix.

If we try to double the number of codes keeping the same value of spreading factor, we obtain the situation depicted in the fig 2(II). Here 64 different codes, each of length 32 is generated and the resulting cross correlation is shown.


Figure 2: Phase Sequences from Perfect Arrays I,II and III

The cross correlation matrix is still mainly diagonal but the perfect orthogonality is lost. Apart from the diagonal, the remaining values of the cross correlation matrix are $1.56 \%$ of the diagonal values.

By doubling again the number of codes of length 32 we obtain the cross correlation matrix shown in fig 2(III). Here we obtain 128 different codes from 32 coefficients.

The price to pay is a higher value of the non-diagonal cross correlation entry, whose mean value is now $3.49 \%$ of the autocorrelation value.

It is interesting, however, how the correlations are distributed along the diagonal value.

This observation suggests that a code assignment policy may improve the performances of a system which is using such a combination of codes.

In a real system the interference between two codes is influenced by several factors, like propagation, position and mobility of the devices, shadowing obstacles and so on.

By using dynamical code assignments policies is possible to take into account some of these factors in a way to reduce sensitively the co-channel interference. The almost diagonal distribution of the highest cross correlation 
values produces an ideal playground where the DCA algorithm can exploit its best performances.

It is important to point out that no other code generation technique is able to give a number of codes which is 4 times the spreading factor $K$ with such a proper cross correlation pattern.

\section{The Asynchronous case}

In this section we analyze the behavior of the PS-CDMA when the signals are received with unexpected delays. Since a synchronization device is supposed to be present, we consider the receiver perfectly synchronized with the main incoming signal of the desired user. By doing that in general the receiver is not synchronized with the interferers and all the delayed replicas of the desired signal due to multipath.

Under these conditions, the ideal behavior of a set of spreading signals $\hat{s}_{i}(t)$ is:

$$
\begin{aligned}
& \operatorname{Re}\left[\frac{1}{T_{b}} \int_{-T_{b} / 2}^{T_{b} / 2} \hat{s}_{i}^{*}(t-\tau) \hat{s}_{i}(t) d t\right]= \begin{cases}1 & \tau=t \\
0 & \text { otherwise }\end{cases} \\
& \operatorname{Re}\left[\frac{1}{T_{b}} \int_{-T_{b} / 2}^{T_{b} / 2} \hat{s}_{i}^{*}(t-\tau) \hat{s}_{j}(t) d t\right]=0 \quad i \neq j
\end{aligned}
$$

with $\tau$ ranging in $\left[-T_{b} / 2, T_{b} / 2\right]$.

In the previous sections we have analyzed the autocorrelation profile (14) for the PS-CDMA signals, showing that the theoretical limit is ideal for $K \rightarrow \infty$.

We now consider a practical case and compare the auto/cross-correlation profiles of both direct sequences and perfect array phase sequences signals. We consider the situation where the same information symbol is continuously repeated by both the desired and the interferent user.

No phase distortions effects are taken into account and a spreading factor $K$ of 32 is considered.

Fig 3 shows the cross-correlation profile (continuous line) and the auto-correlation profile (dotted line) of two spreading signals.

In the upper part of the fig 3, two signals generated by the proposed PS technique are compared. The upper-left part shows the effect of delay on two signals derived by Chu Perfect Sequences. Chu Sequences do not guarantee the perfect orthogonality with $\mathrm{r}=0$, but the delayed crosscorrelation is bounded in the whole range of delay.

On the upper-right part is shown the profile for two signals derived by Perfect Arrays. This time the orthogonality is perfect for the synchronous case $(\tau=0)$ and the delayed profile is extremely low, with the exception of a narrow pulse. Basically the spreading signals derived from a Perfect Array are composed by a discrete cyclic delayed copy of the first sequence.
This may appear as an undesired property, but the narrowness of the impulse is the key issue here. As confirmed by computer simulations, later described, the cross correlation impulse is so narrow that only a few times a multipath replica is likely to fall inside it. This results in a global averaged good rejection of the delayed replicas of the interference signals.
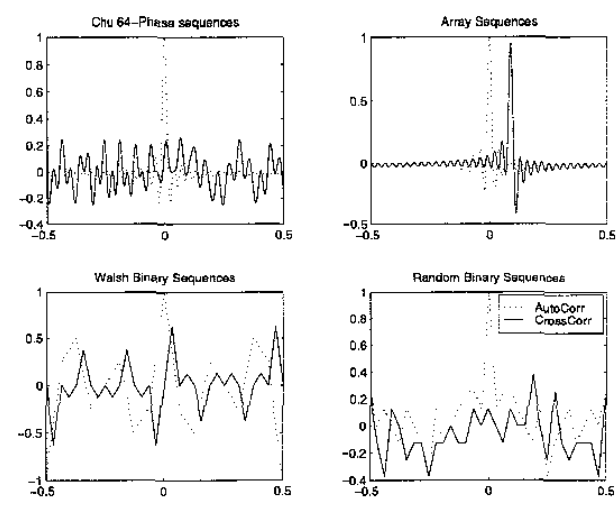

Figure 3: Auto- and cross-correlation profile in the asynchronous case

As the lower half of the fig 3 concerns, the same cross/auto correlation profile is computed for an ordinary Direct Sequences spreading signal. Each chip modulates a square pulse and both Walsh and random binary codes are represented. While orthogonality holds in the synchronous case also for Walsh DS codes, as soon as the signal is delayed, DS signals manifest poor cross and auto correlation profiles.

\section{Computer simulations and results}

To prove the observations stated in the previous sections, several computer simulations have been made.

The general assumptions in common to each simulated environment are:

1. Two users sharing the same channel. One is the desired user whose information is wanted; the other one is the interferer,

2. AWGN noise added to the received signal,

3. Severe multipath channel in the asynchronous case,

4. Performance measured in terms of probability of error at the receiver for the desired user computed for different values of signal to noise ratio.

The CDMA system under test are:

- Direct Sequences CDMA with Walsh codes and rectangular pulse modulated by each chip, 
- Phase Sequences CDMA whose complex codes are obtained by the perfect arrays.

\section{The multipath environment}

We now prove that the unusual cross-correlation profile exerted by the perfect arrays in the PS-CDMA is convenient in the presence of strong multipath. Starting from the GSM channel model, we have developed a discrete simulation environment capable to generate the effect of a strong multipath.

The channel model used in this set of simulations is basically a time-varying complex filter, whose taps are synchronously updated by several pink Rayleigh discrete processes.

The Fig. 4 helps to understand the structure of the channel model. The stream of samples is filtered by a complex Finite Impulse Response filter whose dimension (the parameter WINDOW) infiuences the multipath spread of the channel. A white Gaussian complex process is then added to the resulting signal.

The taps of the complex filter are continuously updated by the Rayleigh generator and spread profiler block.
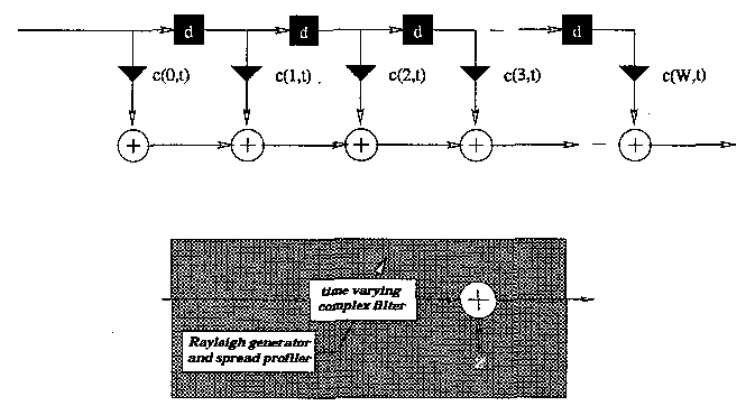

Figure 4: Discrete multipath channel model and Time varying complex filter

Each tap of the complex filter is synchronously updated from a set of independent Rayleigh generators. The structure of each complex tap generator is described in the fig. 5 .

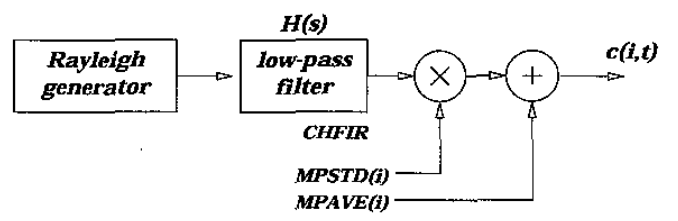

Figure 5: Complex Taps Generation

Each complex tap is generated by a Rayleigh process with unitary variance. This process is then filtered by a low pass FIR used to shape the power spectral density of the process. The FIR taps are defined in the CHFIR vector which is a parameter of the simulation. Basically the CHFIR vector defines the frequency response of the spectral shaping filter $H(f)$. Since the Rayleigh generator is white, the quantity $\|H(f)\|^{2}$ gives the power spectral density of the channel taps. The first and second order statistics of the channel taps are defined separately for each tap by the two vectors MPAVE and MPSTD. Basically the tap $c(i, t)$ is a pink Rayleigh (or Rice) process with variance $M P S T D(i)^{2}$ and mean $M P A V E(i)$.

The two system, DS-CDMA and PS-CDMA, have been tested with different values of the received interferer power. Table 1 shows the scalar parameters used in the simulation, while in the fig. 6 are plotted the values of the vectors involved.

\begin{aligned} & \hline spreading factor 16 \\ & \hline received user power 1 \\ & \hline received interf. power $10,4.65,2.15 \\ &$\hline SNR (desired user) dB $0 \cdots 8 \\ &$\hline FFT size 32 \\ & \hline WINDOW 32 \\ & \hline phase sequences type perfect 32-phase arrays \\ & \hline direct sequences type Walsh 16 \\ & \hline\end{aligned}

Table 1: Asynchronous case - profile A - parameters

In the fig. 7 there is a comparison between a DS-CDMA system and a PS-CDMA one, both facing a severe multipath environment. The performance of the proposed technique is clearly better than the direct sequences. As expected this gap increases as the AWGN noise is reduced. The cross-correlation properties of the Phase sequences are useless when the dominant cause of error is the AWGN noise.

\section{Conclusions}

In this paper we proposed an alternative technique for the design of spreading signature waveforms to be used in Code Division Multiple Access systems. We presented two methods for the code design; the first one achieves a substantial increase in the code space and an easy implementation. The second one allows the use of a special class of complex codes named "perfect $\mathrm{N}$-phase arrays" whose cross-correlation properties are found useful to fight the effect of multipath. Computer simulations confirm the theoretic analysis of the proposed waveforms.

An easy FFT implementation of a PS-CDMA transmitter and receiver is also also suggested. Common and lowcost DSP blocks are used in the implementation of the devices.

At the time of writing the impact of multiuser technique on the performances has not been investigated. Moreover, in the analysis we considered a flat channel, with no am- 

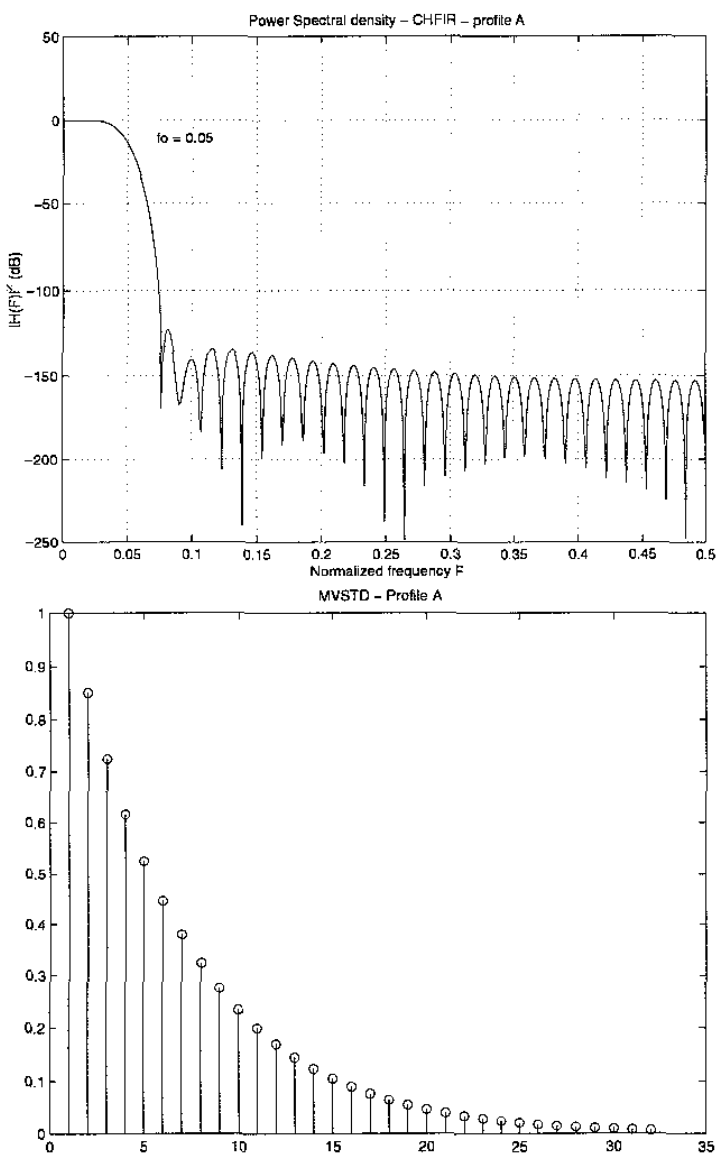

Figure 6: Profile A - vector parameters

plitude distortions over the bandwidth occupied by the signals. One of the future investigation tracks will be the design of Phase Sequences with non-flat spectral shape. This will be achieved simply removing the modulus constraint on the spectral coefficients.

Adaptive algorithms will be also considered to improve the orthogonality of the signals, and to reduce the linear phase distortion induced by a time delay in the reception of a PS-CDMA waveform.

\section{References}

[1] L. Bömer and M. Antweiler. Perfect n-phase sequences. IEEE Journal on Selected Areas in Communications, 10(4):782-789, May 1992.

[2] D. C. Chu. Polyphase codes with good periodic correlation properties. IEEE Trans. Inform. Theory, IT18:531-532, 1972.

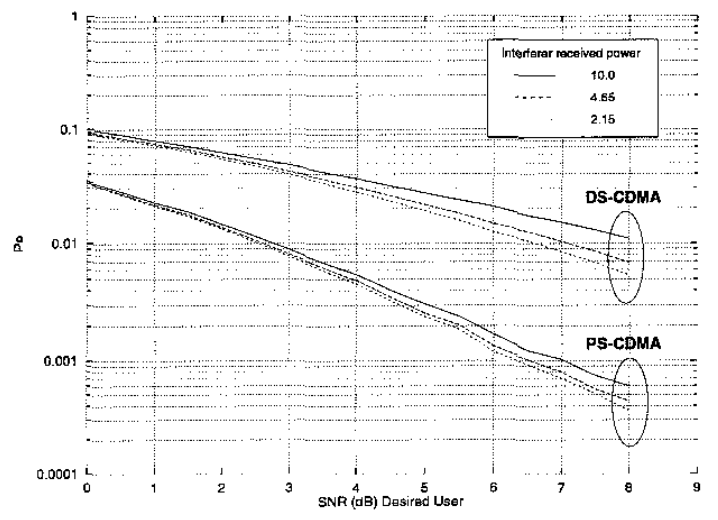

Figure 7: PS-CDMA and DS-CDMA in the asynchronous case

[3] R. L. Frank and S. Zadoff. Phase shift pulse code with good periodic correlation properties. IEEE Trans. Inform. Theory, IT-8:381-382, 1962.

[4] A. Milewski. Periodic sequences with optimal properties for channel estimation and fast start-up equalization. IBM J. Res. Develop., 27:426-431, 1983. 